\title{
Dinâmica dos fluidos aplicada ao estudo da halocinese
}

\author{
Cordeiro, C. H. T. ${ }^{1^{*}}$; Oliveira, L.G.S. ${ }^{1}$
}

1 Programa de Pós-Graduação em Energia, Universidade Federal do Espírito Santo, São Mateus, ES, Brasil. 2 Departamento de Ciências Naturais, Universidade Federal do Espírito Santo, São Mateus, ES, Brasil.

* e-mail: kayquethomazini92@gmail.com

\begin{abstract}
Resumo
A busca por hidrocarbonetos se inicia no conhecimento do ambiente à que o petróleo esteja submetido, podendo encontrar certos tipos de barreiras que devem ser evitadas ou redirecionadas, como os diápiros de sal. A formação de domos salinos é muito mais complexa do que pressupunham os modelos clássicos, sendo dependente não somente da sobrecarga sedimentar, mas também pode ser iniciada por esforços extensionais ou compressionais, isto é, a tectônica da bacia é tão importante quanto a sedimentação para iniciar um movimento salífero. O presente trabalho visa o estudo da halocinese, devido ao sal possuir características que em determinadas condições de pressão e temperatura, tanto em superfície, quanto no interior da Terra, formam tal estrutura. Como o sal se comporta semelhante ao fluido [5], as equações de Navie-Stokes e bi-harmônica serão utilizadas, sendo feito uma simulação numérica e uma modelagem computacional utilizando o software MATLAB (MATrixLABoratory).
\end{abstract}

\begin{abstract}
The search for hydrocarbons starts by getting knowledge of the environment which the petroleum is subjected, it may find certain types of barriers that must be prevented or redirected, as the salt diapirs. The formation of salt domes is much more complex than assumed by the classical models, being dependent not only on the overload sediment, but it also can be initiated by extensional or compressional efforts, it means, the tectonics of the basin is as important as the sedimentation in order to start a saliferous movement. This present project aims to study the halokinesis due to salt characteristics that under certain conditions of pressure and temperature, both in surface and inside Earth, form such structure. As the salt behaves similar to the fluid [5], the Navie-Stokes and Biarmonich equations will be used, being that, a numerical simulation and computational modeling will be processed by the MATLAB software (MATrixLABoratory).
\end{abstract}

Keywords: halokinesis, Navie-Stokes, Matlab.

\section{Introdução}

O fenômeno da halocinese envolve a movimentação de rochas evaporíticas, por meio da ação gravitacional, podendo gerar intrusão e deformação de camadas adjacentes e sobrepostas, formando assim o que são chamados de corpos de sal.

A formação de domos e diápiros de sal é muito mais complexa do que pressupunham os modelos clássicos, sendo dependente não somente da sobrecarga sedimentar, mas também pode ser iniciada por esforços extensionais ou compressionais, isto é, a tectônica da bacia é tão importante quanto a sedimentação para iniciar um movimento salífero [3].
A instabilidade gravitacional do fluido mais pesado sobreposto no fluido mais leve é conhecida como instabilidade de Raylegh-Taylor, o tempo de crescimento é uma função que determina quando irá começar essa instabilidade e fatores importantes como a densidade, espessura das camadas salina e sedimentar, a viscosidade do sal auxilia para encontrar esse início da instabilidade.

A revisão bibliográfica realizada no estudo, discorre sobre as bacias brasileiras e as ocorrências de diapirismo. A equação de Navier-Stokes para fluidos viscosos é apresentada na Equação 1, e a equação biahmônica é apresentada pela Equação 2. Com as operações matemáticas adequadas [4], chega-se a 
Equação 4 que mostra o tempo necessário para o início da instabilidade de Railegh-Taylor de um diápiro de sal.

A equação básica para momento diferencial para um elemento infinitesimal [4] é:

$$
-\nabla+\nabla .=
$$

Utilizando a equação da continuidade que é apresentada pela Equação 2, lembrando que esta equação vale para um escoamento incompressível e a função da continuidade é definida por , eliminando a pressão na Equação 3 de b̉alanço de força em duas dimensões tem-se a equação bi-harmônica, em termos do operador Laplaciano $\nabla^{2}$

$$
\begin{aligned}
& \nabla \cdot \mathrm{v}=0 \\
& \nabla^{2}=\quad \mathrm{L}^{2}+\mathrm{L}^{2}
\end{aligned}
$$

Para o fluxo em duas dimensões um fluido muito viscoso a função da continuidade satisfaz a equação bi-harmônica. A função da continuidade tem uma interpretação física em termos da taxa volumétrica entre dois pontos em um fluxo incompressível, permanente e de duas dimensões. Com essas equações, utilizando de operações matemáticas a partir de condições iniciais, chegamos a equação do tempo de crescimento [4]:

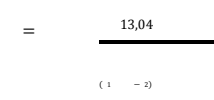

O tempo de crescimento é dado pela Equação 4, que é dependente da viscosidade, da diferença entre as densidades da gravidade e também da espessura.

Para melhor estudo do tempo de crescimento foi utilizado o conceito do método dos elementos finitos (FEM), utilizando a formulação fraca feita por Galerkin.

\section{Simulação, Resultado e Discussão}

O estudo utilizou três casos onde será estudado o diapirismo de domos de sal, que são eles: o tempo de crescimento, viscosidades iguais, e pelo contraste de viscosidade.

\subsection{Tempo de Crescimento}

Para visualizar o tempo de crescimento um algoritmo foi utilizado baseado na equação 4 , com isso varia-se a densidade de três tipos de rochas sedimentares com a rocha salina utilizando a Tabela 1, mantendo a viscosidade do sal conforme mostrado por [6] que é $10^{14}$ Pa.s, e a Tabela 2 apresenta a espessura de cada camada.
Tabela 1: Densidades da camada sedimentar e da camada salina para $\underset{\substack{\text { trens } \\ \text { Dendsoses }}}{\text { tipos de combinações }}$

$$
\text { Sed. }\left(\mathrm{kg} / \mathrm{m}^{3}\right) \quad\left(\mathrm{kg} / \mathrm{m}^{3}\right)
$$

\begin{tabular}{rlll}
\hline Tipo 1 & 2400 & 2200 & 200 \\
\hline Tipo 2 & 2500 & 2200 & 300 \\
\hline Tipo 3 & 2600 & 2200 & 400 \\
\hline
\end{tabular}

Tabela 2: Espessura da camada sedimentar

\begin{tabular}{cc}
\hline & Espessura \\
\hline Tipo 1 & 2000 \\
\hline Tipo 2 & 5000 \\
\hline Tipo 3 & 9000 \\
\hline
\end{tabular}

Na Figura 1 tem-se o gráfico semi-log para melhor visualização do tempo de crescimento versus a espessura da camada sedimentar pode-se observar que quanto maior for à diferença de densidade ( $1-2$ ), mais rápido tem-se o tempo de crescimento, lembrando que o tempo de crescimento é o tempo necessário para que a instabilidade na camada se inicie e é dado em segundos. Também é possível observar que quanto maior a espessura da camada também mais rápida o tempo de crescimento, isso acontece porque é considerado o campo de pressão, ou seja, quanto maior a camada e maior a diferença de densidade $(1-2)$, mais rápido se iniciará a formação de um diápiro.

O mesmo vale para a Figura 2, de novo tem-se o gráfico semi-log para melhor visualização, do tempo de crescimento versus a diferença de densidade e agora se varia a espessura da camada sedimentar. Pode-se observar que quanto maior a espessura da camada mais rápida será o início da instabilidade.

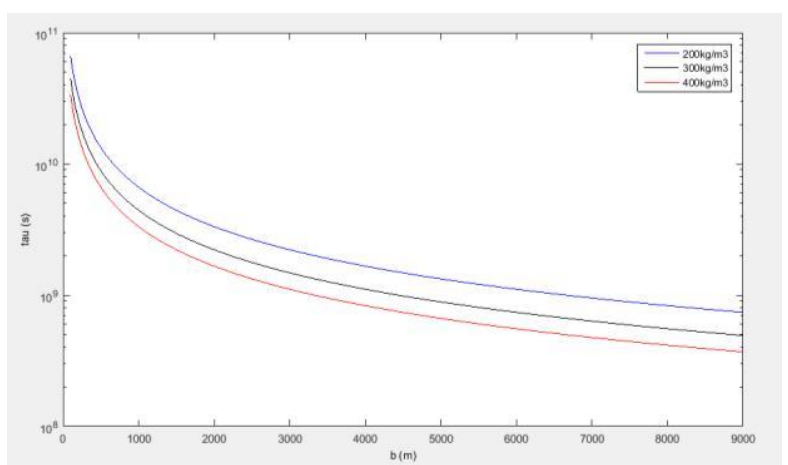

Figura 1: Tempo de crescimento em segundos versus a espessura da camada sedimentar em metros variando a densidade da carga sedimentar. 


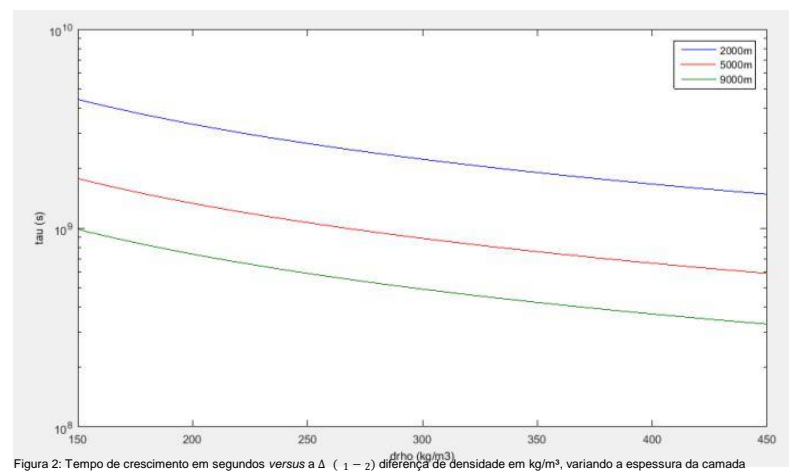

sedimentar.

\subsection{Isoviscoso e viscosidade diferente}

Na situação de isoviscosidade o método dos elementos finitos utilizando a formulação fraca de Galerkin mostrada por [1], será utilizado no Matlab (MATrixLABoratory), com uma geometria retangular como mostrado na Figura 3:

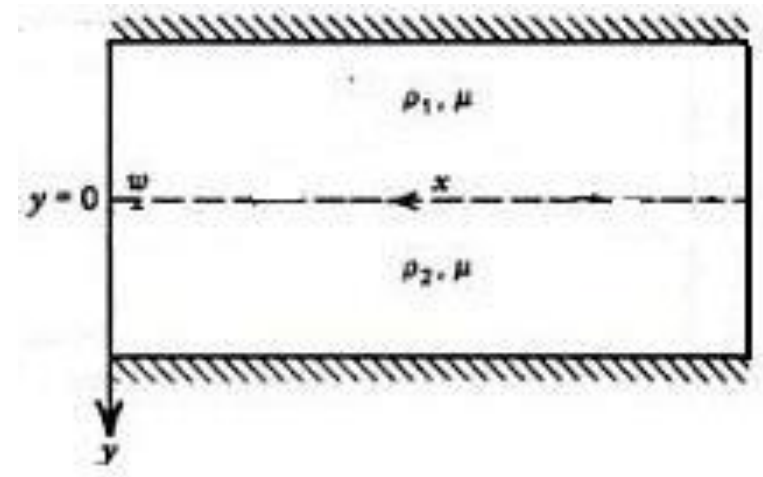

Figura 3: Geometria retangular utilizada para a simulação do caso isovisco e com contraste de viscosidade.

É possível observar na figura que ${ }_{1} e_{2}$ que para gerar a instabilidade, a densidade da rocha de cima deve ser maior que a de baixo entẫo, $(1>2)$ essa propriedade deve ser atendida [1].

Também vale lembrar que foram eliminados dos cálculos a influência da temperatura e eventuais tensões cisalhantes como compressão ou distensão que possam ocorrer na bacia, tem-se que apenas o campo de pressão será utilizado para esse tipo de situação.

Com isso, comparando com o que acontece naturalmente para a formação de diápiros, vale considerar que 1 será uma rocha sedimentar qualquer e 2 uma rocha salina, os valores das densidades utilizados foram o mesmo da Tabela 1 do tipo 2, já a viscosidade nessa forma isoviscosa será igual e adimensionalizada de valor $=(1 \mathrm{e} 4) / 30$ [6], foram 100 passos de tempo até a conversão do modelo:
No caso das viscosidades diferentes utilizou-se o mesmo método (FEM) e a mesma geometria da Figura 3, porém agora com 0 contraste de viscosidade, com valores adimensionais, como é comparada com casos reais, a viscosidade do sal é maior, cerca de cinco vezes a viscosidade de outra rocha sedimentar (uma média), com isso, os valores utilizados foram: $1=(1 \mathrm{e} 4) / 30$ e $2=$ $(0.2 \mathrm{e} 4) / 30$, já as densidades continuaram diferentes e as mesmas utilizadas na Tabela 1 do tipo 2 , foram 60 passos de tempo até a conversão do modelo, foram 60 passos de tempo até a conversão do modelo.

Com isso foi possível a modelagem de um diápiro de sal que aparece na Figura 4 e pode-se observar a forma semelhante ao de um cogumelo por ela gerada devido a condição de isoviscosidade do modelo estudado. Na Figura 5 mostra uma figura semelhante ao de uma muralha sendo a parte inferior e a superior de espessuras semelhantes isso se dá ao fato da diferença de viscosidade ao modelo estudado.

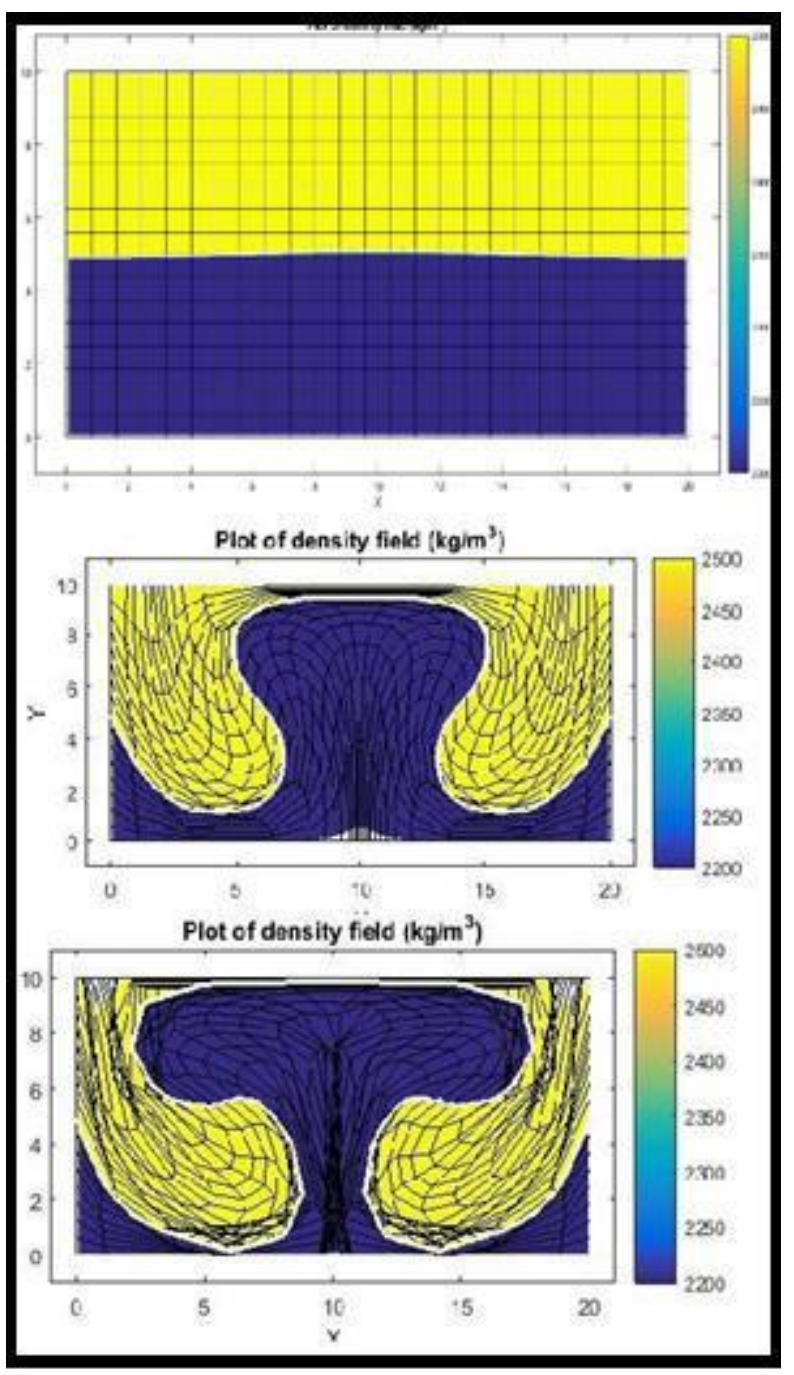

Figura 4: Simulação da formação de diápiros de domos de sal possível a partir da ação gravitacional em condições isoviscosas. 
É possível observar uma grande semelhança dos modelos gerados com a geometria real dos domos salinos encontrados, mostrado na Figura 6 e comparados com a Figura 4 e a Figura 5 em seções sísmicas. Lembrando que esse trabalho utilizou-se basicamente as diferenças de viscosidade e densidade dos fluidos em questão, bem como o tempo de ocorrência dos fatos.

Pode-se observar que no estágio intermediário de formação em ambos os casos, a geometria é muito semelhante. Portanto, pode-se concluir que, conhecendo as propriedades dos fluidos em questão, pode ser feita uma previsão de como aquele domo salino irá se comportar com o passar dos anos, ou seja, poderá ser conhecida sua geometria depois de milhões de anos.

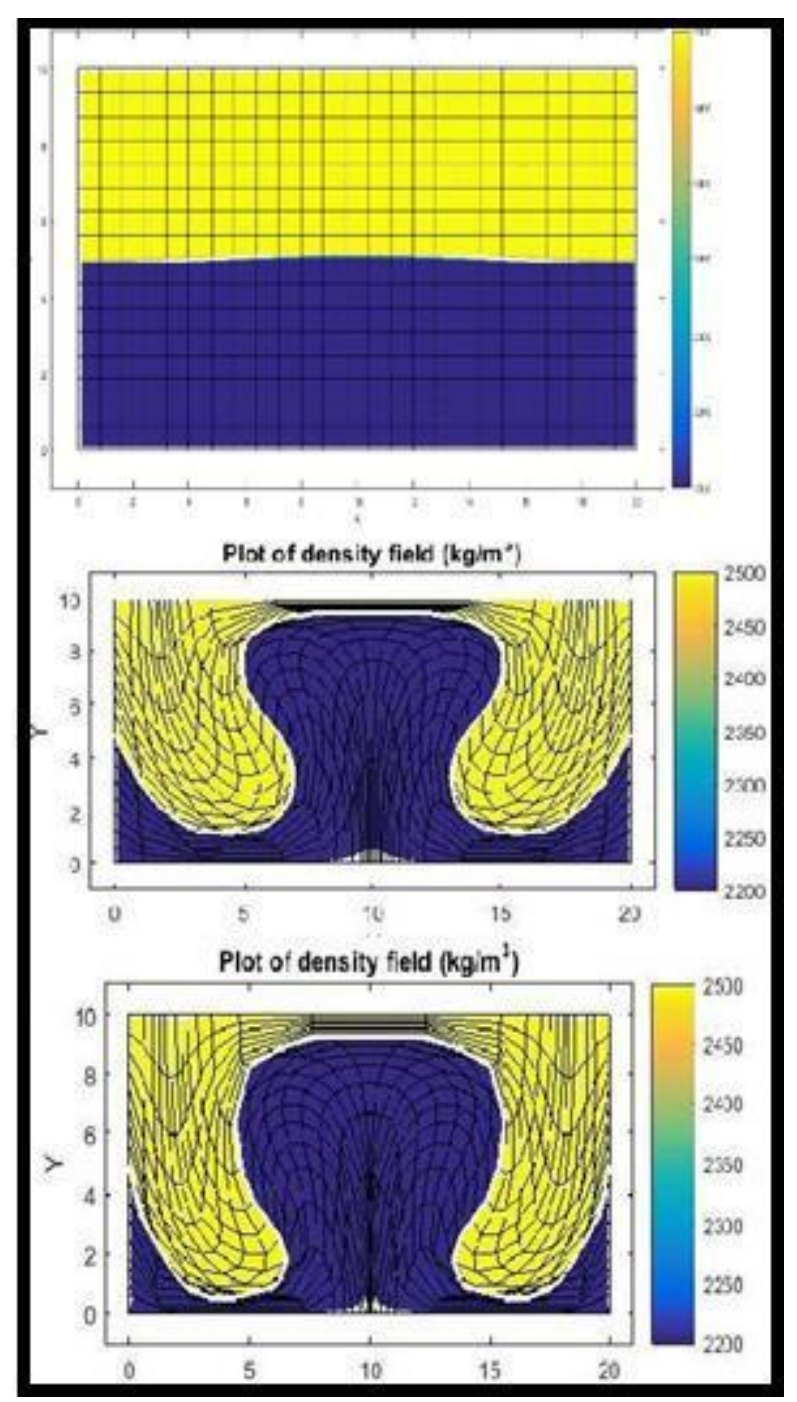

Figura 5: Simulação da formação de diápiros de domos de sal possível a partir da ação gravitacional em condições de diferentes viscosidades.

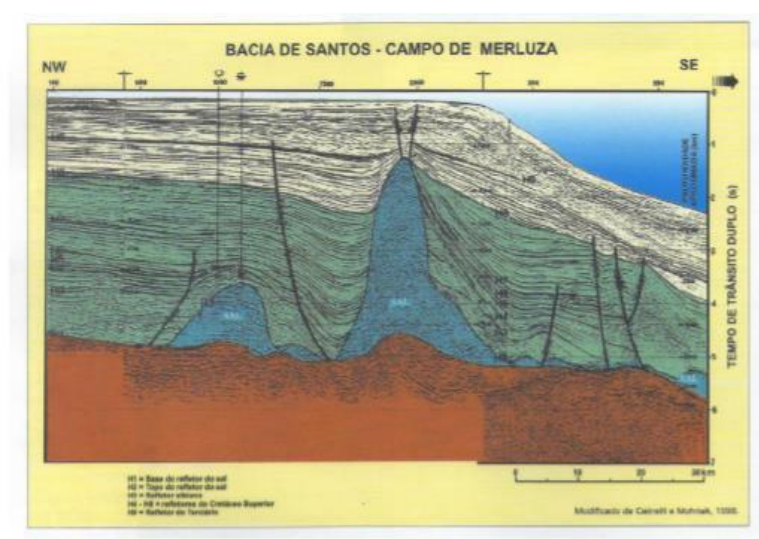

Figura 6: Seção sísmica mostrando a tectônica de sal na área do campo de Merluza [2].

\section{Conclusão}

A formulação e a sua aplicação em um programa computacional MATLAB (MATrixLABoratory) foi aplicado nesse trabalho, sendo que essa instabilidade só acontecerá se o fluido da camada superior for mais denso que o fluido de camada inferior, isso normalmente acontece na formação de diápiros de domos de sal, então a aplicação dessa função do tempo de crescimento é muito válida para prever quando a instabilidade se dará início.

Depois disso para ser encontrada uma melhor aplicação dessa função utilizamos o método dos elementos finitos, onde a partir disso foi possível aumentar as informações sobre domos salinos.

\section{Nomenclatura}

- Densidade;

g - Gravidade;

FEM - Método dos Elementos Finitos;

b - Posição;

P - Pressão;

$\mathrm{t}$ - Tempo;

- Tempo de crescimento;

- Tensão de cisalhamento;

- Viscosidade

\section{Referências Bibliográficas}

[1] ASSAN, A.E., Método dos elementos finitos Primeiros passos. Campinas, SP: Editora da Unicamp,2003. 
[2] CAINELLI, C.; MOHRIAK, W. U. Geology of atlantic eastern Brazilian basins. In: AMERICAN

ASSOCIATION OF PETROLEUM GEOLOGISTS.

INTERNATIONAL CONFERENCE AND EXHIBITION SHORT COURSE, 1998, Rio de Janeiro. [Procedings] [S.1 : s.n.], 1998. Bazilian Geology part II, 67 p.

[3] CASTRO, A. S.; HOLZ, M. A tectônica de sal e a deposição de sedimentos em águas profundas na região sul da Bacia de Santos. In: IBP III Congresso Brasileiro de P\&D em Petróleo e Gás, Salvador, 2005), 2005.

[4] MOHRIAK P.; SZATMAN W.; ANJOS S. M. C.; Sal: Geologia e tectônica. 1. ed. [S.I.]: BECA, v. 1, 2008.

[5] TRUSHEIN, F. Mechanism of salt migration in northen Germany. American Association of Petroleum Geologist Bulletin, Tusla, Okla., v . 44, n. 9, p. 15191540, 1960.

[6] TURCOTTE, D.; SCHUBERT, G. Geodynamics.

[S.I.]: Cambridge University Press, 2002. 